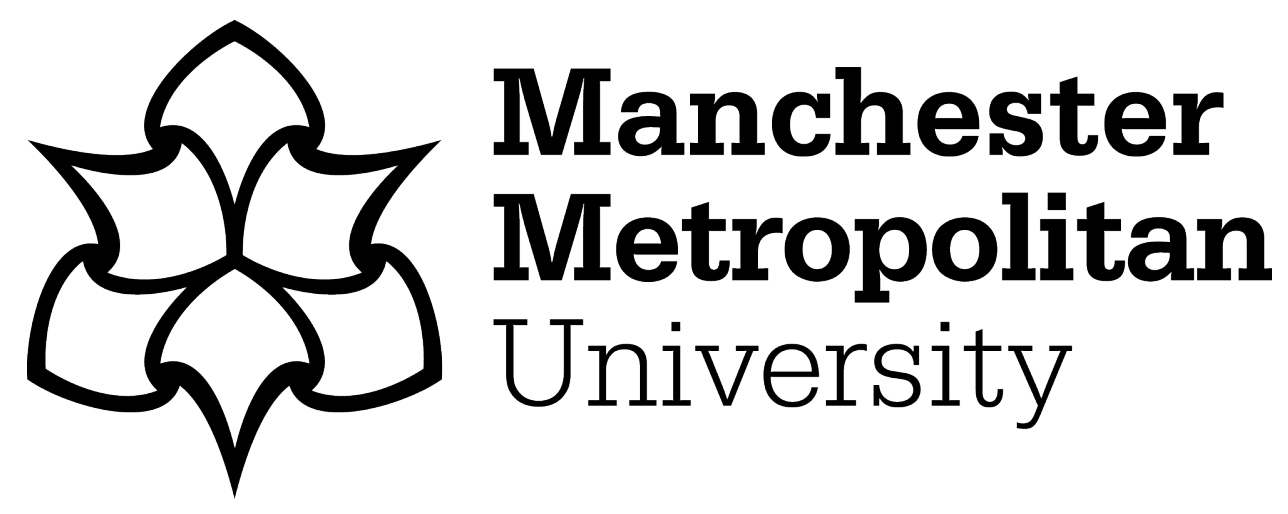

Pearson, Emma, Byrne-Davis, Lucie, Bull, Eleanor ORCID logoORCID: https://orcid.org/0000-0002-9444-0597 and Hart, Jo (2020) Behavior change techniques in health professional training: developing a coding tool. Translational Behavioral Medicine, 10 (1). pp. 96-102. ISSN 1869-6716

Downloaded from: https://e-space.mmu.ac.uk/622970/

Version: Accepted Version

Publisher: Oxford University Press (OUP)

DOI: https://doi.org/10.1093/tbm/iby125

Please cite the published version 


\title{
Manuscript Title: Behaviour change techniques in health professional training: developing a coding tool
}

\begin{abstract}
Background

Health professional Continuing Professional Development (CPD) courses often aim to change practice; understanding which training techniques drive behaviour change can help educators facilitate this. The 93-item Behaviour Change Technique Taxonomy (BCTT) describes behaviour change techniques (BCTs) used in behaviour change interventions but was not designed for understanding CPD; it is necessary to explore how best to use the BCTT in this context.
\end{abstract}

Purpose

This study aimed to explore the BCTs used by CPD course educators to change healthcare practice and to develop and pilot an e-tool, based on the BCTT, to enable course designers and educators to understand which BCTs are in their training. This understanding could lead to enhanced CPD and an experimental approach to assessing the benefits of including a variety of BCTs in CPD.

\section{Methods}

Two psychologists, trained in using the BCTT, observed three postgraduate medical CPD courses. In phase one, the BCTT was used to code 26 hours of observations. An e-tool including observed BCTs was developed and used to code 35 hours of observations in phase two. Feedback was collected through short discussions with educators from each course. The tool was further refined in phase three. 
$\underline{\text { Results }}$

37 BCTs were identified in phase one, a further four in phase two and a further two in phase three. The final e-tool comprised 43 BCTs with examples of their use based on course observations to aid identification, since educators fed back that they would value an uncomplicated tool with practice-related examples.

\section{$\underline{\text { Conclusions }}$}

A coding tool to understand the active ingredients in health professional CPD could enable educators to maximise the impact of CPD on practice. Further work should explore whether educators themselves are able to use the tool to code their training interventions.

Key words: Continuing Medical Education; Professional Development; Curriculum Development/ Evaluation

\section{Introduction}

Health and social care practitioners are concerned with the health, safety and wellbeing of patients, and, in most countries, are subject to regulators who require evidence of regular Continuing Professional Development (CPD), or Continuing Medical Education (CME), to promote continual learning and development to practise safely and effectively $[1,2]$. CPD courses aim to improve practice and thereby the patient experience, often focusing on changing practice behaviour in-line with current developments in evidence-based practice. In the UK, CPD is often the primary solution when practice change is needed, and often aligns with targets set in response to publication of new clinical guidelines, following specific local or national incidents, which highlighted substandard practice, or with policy drivers such as the National Health Service (NHS) England Five Year Forward View [3]. It is crucial to 
understand ways in which CPD can be made effective and efficient in driving recommended healthcare professional practice change.

Healthcare professional practice can be thought of as a series of complex behaviours, but the assessment of training often falls short of assessing behaviour or its complex determinants. Miller's pyramid [4], often used in education to describe levels of assessment from 'knowing', 'knowing how', through 'showing how' to 'does', implies a direct relationship between capability ('showing how') and doing. In behavioural science, however, knowing, knowing how and showing how are all assessments of 'capability' which is just one of three key determinants of practice; the other two being opportunity and motivation [5]. The processes by which knowledge and skills become used in practice and the factors that govern whether 'shows how' ever become 'does' i.e., opportunity and motivation, are rarely used to evaluate the efficacy of training and rarely stated as the focus of education and training. Skilled educators do often intuitively focus on opportunity and motivation for practice change within CPD courses, such as including a clinical case illustrating the need for change and perhaps research evidence highlighting improved outcome with changed practice. An underlying theory of how behaviour changes and the techniques necessary for achieving meaningful behaviour change in professional practice is implicit. However, because these intended outcomes are not explicit, nor the techniques selected to target explicit outcomes, the extent to which behaviour change techniques are implicitly used in CPD activities is not quantified, nor their efficacy tested.

There is evidence to suggest that health service interventions lacking in theoretical underpinning can hamper the translation of research into practice, affecting the design, success, replicability and improvement of interventions [6]. Furthermore, interventions which are lacking in comprehensive description also impact negatively on replicability, evaluation, implementation and improvement [7]. If we accept that healthcare professional practice is 
made up of a series of complex behaviours, with CPD being a key type of behaviour change intervention, it follows that developing a greater understanding of the theory, methods and techniques involved in complex behaviour change could facilitate the design and effectiveness of CPD. Considering training interventions in relation to the BCTs (which are the basic 'active' ingredients of an intervention) present, we argue, will add an extra dimension to how we characterise CPD training interventions and would go some way to addressing the deficit in comprehensive intervention descriptions and therefore support improvement in quality [7].

Behavioural scientists have taken a theory-based approach to behaviour change, developing several frameworks to support the classification and characterisation of healthrelated interventions, based on their mode of action, to guide intervention development [6]. For example, in addition to the creation of an overarching framework of behaviour describing capability, opportunity and motivation [5], the active content of interventions has been codified in the Behaviour Change Technique Taxonomy (BCTT) [8]. This consists of 93 irreducible, stand-alone 'active ingredients' of interventions with the potential to change behaviour, alongside their definitions and one or two examples of their application. Some techniques focus more on the motivation drivers of behaviour, others on opportunity and building capability.

The BCTT has a generic focus and only 12 of the 104 examples relate to health professional practice behaviours. Equally, in the many studies published using the taxonomy, it has been frequently applied to designing, describing and evaluating behaviour change interventions for the general public or patients, often to change healthy living or medication adherence behaviours [e.g. 9, 10], and less frequently applied to interventions to change health professional practice [e.g. 6]. Furthermore, some techniques may not be easily applicable to a training context, such as 'biofeedback', where people would need to be given 
feedback on their body state using an external monitoring device to try to influence their practice. The BCTT is, therefore, likely to be somewhat over-comprehensive in relation to education, which may explain why it has rarely been used to code health professional education and CPD activities $[11,12]$ as they are delivered: i.e., 'live coding'. We propose that if educators could describe the content of education interventions in terms of BCTs, this would facilitate investigations of the efficacy of elements of CPD programmes. Knowing what is present in training could then open a research space to explore whether there might be ways of improving CPD interventions. Research supports the utility of the BCTT framework as a means of guiding behaviour change intervention [6]; knowing more about behaviour change principles could enable educators to create CPD courses which are more effective and increase the impact of training on practice.

We aimed to adapt the BCTT, focusing on the techniques that are common in health care professional CPD education, with relevant examples, to create a tool that was feasible to use to live code CPD. Specifically, we aimed to identify, from the 93 techniques in the $\mathrm{BCTT}$, the techniques that could or are likely to be present in high quality healthcare professional CPD; to create a simple coding e-tool with relevant training examples and explore if it can be used to understand and code techniques observed on a range of example medical CPD courses aiming to change healthcare practice. This tool could then be utilised by course developers and educators to facilitate theory-driven, evidence-based development of their courses to optimise the impact of the behaviour change goals of their CPD courses. Being able classify which BCTs are included in CPD training courses will add to the theoretical exploration of what works, how it works and for whom does it work best, as well as in the practical design of behaviourally-focussed CPD training. 


\section{Methods}

This observational study involved three phases of data collection. Phase one involved observing the face-to-face teaching elements of three medical CPD courses to identify those BCTs in the BCTT used to change practice behaviour. We observed three courses to see a range of course and tutor. Courses were developed by two different medical CPD organisations in three different clinical contexts - adult acute illness, acute obstetric emergencies and acute paediatric emergencies delivered by a range of specialist educators including doctors of different specialities and nurses. In phase two, a coding tool was created and piloted through further observation of the three courses; in the third phase, final refinements were made (see figure 1 for summary of methods).

\section{Phase 1}

Two observers (X and Y), trained in using the BCTT, attended three medical CPD courses together; courses were selected based on their differing clinical topics and enrolment of multi-disciplinary professionals at different specialties/grades/backgrounds. Course A was a one-day course focussed on recognising and responding to the deteriorating patient in hospital settings; course B was a two-day course focussed on helping professionals recognise and manage life-threatening illness and injury in children; course $\mathrm{C}$ was a two-day course that helped professionals apply a structured approach to recognise and manage maternal and foetal severe illness and injury. Courses were observed across multiple training sites in the North West of England, UK. Phase one involved coding course A, and day two of courses B and $\mathrm{C}$, with a total of 26 hours of observation.

Course A facilitators were critical care practice-based practitioners and educators, whilst course B and C facilitators were medical professionals selected by the over-arching course providers after having performed exceptionally well when participating in the course 
themselves. Observed course days were delivered by different sets of facilitators, working to a course manual for each course and an agreed set of tutor notes and materials. Teaching methods included collaboration between course participants, the use of actual medical equipment and technology to demonstrate practice behaviour and for participants to practice behaviours, and a mixture of small and large group teaching and learning. Courses were typically attended by 15-25 participants, the majority of whom were doctors of various grades (commonly anaesthetists, gynaecologists, accident and emergency specialists and paediatricians); others were acute nurses and midwives.

Independent live-coding of the face-to-face teaching on each course was completed on paper using the BCTT to map the techniques used and their frequency. Observers coded BCTs which were present beyond doubt and were perceived as being 'core' to the course (i.e. considered to be a key focus of the training) or 'occasional' (i.e. considered to be important but more peripheral in focus) in terms of their prevalence, along with BCTs which were apparent, yet not quite fully delivered (coded as 'missed opportunities') or absent where not observed at all. Inter-rater reliability of the coding was then assessed using Cohen's Kappa and the prevalence adjusted bias adjusted kappa (PABAK) [13].

\section{Phase Two}

The research team worked together to structure the observed BCTs into an e-tool and refinements were made (e.g. helpful descriptions were added to facilitate coding). BCTs that were not coded during the initial 26 hours of observations were added based on the expertise of the research team and in consultation with six trained BCTT coders with expertise in observing the practice change elements of healthcare CPD.

The agreed BCTs were then structured into a coding tool based on the principles of other action coding frameworks (for example, Dementia Care Mapping [14]) using local 
software that allowed development of an e-tool on both app and website format (Z). To test its feasibility, $\mathrm{X}$ and $\mathrm{Y}$ observed a further 35 hours of the face-to-face teaching involved in the CPD courses, using the e-tool to code BCTs. This involved coding one further delivery of course A, both days of course B and day one of course C; inter-rater reliability was assessed. Further feedback on the e-tool and its potential value was sought through brief discussions with one facilitator from each course day. Discussions included what the course facilitators perceived as the behavioural targets of their course, their views on the aesthetics and content of the e-tool, and how useful they may find the e-tool in practice. Field notes were recorded during these discussions, and the themes were discussed during team meetings to inform refinements.

\section{Phase Three}

The coding framework was refined to incorporate the BCTs observed during both phase one and phase two. Examples were developed for BCTs coded throughout the 61 hours of observed CPD, including examples of a 'missed opportunity' for each BCT. Examples were based on observations made during the CPD sessions and so were designed to help educators to easily identify each BCT, along with examples of the missed opportunities that might occur where a facilitator did not quite meet the requirements of the $\mathrm{BCT}$ as defined by the BCTT [8]. Examples were discussed and refined by the research team with helpful feedback from an additional five psychologist coder colleagues with experience in coding live health professional CPD courses. In refining the examples, the team and additional experts consulted commented on whether they thought any BCTs might be missing from the framework, and the relevance and accessibility of examples.

In conjunction with experts in the field of behavioural science, guidelines for validating BCT examples were developed, including the assessment of an example's 
accuracy, distinctiveness from other BCTs, clarity and generalisability. A team of seven independent UK-based health psychology researchers from a university health psychology group used this tool to rate a sub-section of the examples $(\mathrm{N}=9)$ and three members of the research team $(\mathrm{X}, \mathrm{A}, \mathrm{B})$ used it to each rate one-third randomly chosen examples of those that remained; $10 \%$ of examples were double-coded.

Following this feedback, further refinements were made to the framework and a final version produced.

\section{Results}

\section{Phases One and Two}

Three CPD courses were observed (a total of 61 hours), using the complete 93-item BCTT as a framework to live-code courses in action in phase one. An e-tool was created following phase one observations to live-code course content in phase two. Inter-rater reliability was high: Cohen's Kappa scores fell between 0.75 and 0.89 and PABAK between 0.81 and 0.92 (see table 1).

In phase one, 15 BCTs were identified as core, 22 as occasionally used, and six as missed opportunities; 56 were absent. In phase two observations, 17 BCTs were identified as core, 31 as occasionally used, one as a missed opportunity.

Taking phase one and two together, a total of 39 BCTs from BCTT v1 [8] were identified as present (either core or occasionally used) over the three courses. Figure 2 displays those coded in course A, B and C separately. There were 10 'missed opportunity' BCTs, eight of which were also fully used elsewhere in the course. Figure 3 displays the missed opportunities coded in course A, B and C separately. The remaining BCTs from 
BCTT v1 [8] were absent from phase one and two observations and were not included in the e-tool. Following discussion and expert opinion described in the methods, two further BCTs were added in phase three (see appendix S1 for each of the final 43 techniques included in the final BCT training tool, including rationale).

\section{Feedback from course trainers}

Course educators endorsed the behavioural focus of their courses: one trainer commented that 'it's all about changing behaviour or embedding skills really'. Educators agreed the tool would be useful for understanding their course content and suggested that concrete examples were helpful so that educators could be clear on how they're used. They suggested formatting changes such as reducing text, adding colours and increasing space, and that guidance of how to identify a behaviour would be useful.

\section{Phase Three}

To rate the BCT examples, four criteria were developed (see appendix S2). Members of the research team judged 72 of the 86 examples to meet all four criteria, with 14 deemed as needing further refinement (mostly relating to distinctiveness from other BCTs) and amongst those double screened, inter-rater agreement of acceptability of examples was $81.94 \%$.

A simplified, off-line version of the final BCT training taxonomy e-tool, is in appendix S3. 


\section{Discussion}

We aimed to develop a coding tool including BCTs that are common in health care professional CPD training, with relevant examples. Our findings suggest that it is possible to understand and reliably code the active content focussed on changing practice behaviours used by educators in healthcare CPD courses. We have identified 43 BCTs used in healthcare CPD. We have developed a framework for creating examples of BCTs and, using this framework, have written 72 examples of BCTs that might be observed in education courses to assist capture of and differentiation between BCTs.

There were some limitations to our work. Firstly, whilst this study took a robust approach to data collection, observations and testing of the e-tool occurred with a very small sample of CPD courses in one region of the UK. To address the feasibility and generalisability of the tool, further testing is currently underway using additional courses in the UK, Canada and Tanzania. Secondly, psychologists undertook the observation and coding in this study, both of whom were previously trained to code behaviour change using the BCTT. Therefore, further work is necessary to ascertain how 'user-friendly' the tool is for educators that may not have a background in behavioural science and to identify what training and support may be necessary for its effective use.

Nevertheless, there are many potential uses of this tool. Firstly, researchers can compare different techniques used by different educators, as we have shown it is possible to use the tool to live code CPD reliably. Secondly, exposing the BCTs in CPD education allows for their inclusion in educator training and education manuals. This will enable consistency across distributed learning teams and a means of providing feedback to course educators regarding their inclusion of effective BCTs and thus facilitate educator development. Exposure of BCTs will also allow the systematic variation and testing of their 
efficacy. Whilst not within the scope of this study, future research could evaluate variation in effectiveness of BCTs in CPD education in stimulating sustained change in practice behaviours to ultimately enhance course effectiveness and efficiency. Thirdly, our experience was that educators were keen to understand BCTs they often used implicitly, including feedback on BCTs used instinctively, and 'near misses' that they could enhance with minor changes to their approach or language. We noted that for some educators, knowledge of BCTs appeared to provoke reflection on action [15] and for some teams sparked conversations about course revision and educator training. The act of coding and reflecting back the codes to the educators, therefore, might be an intervention itself, promoting reflection and quality improvement of educators.

\section{Conclusions}

The purpose of health professional CPD education is often to change practice behaviour. Despite this, CPD is typically framed around educational theories and practices which may or may not have behaviour change at their core. This can lead to missed opportunities in influencing factors that might lead to healthcare professionals making prolonged changes to their practice behaviours following training.

Creating a BCT coding tool, which is tailored to healthcare professional practice, we argue, will empower healthcare education providers to understand the different behaviour change ingredients used in healthcare CPD. This will ensure courses are of high quality and aimed at driving sustained practice change in-line with the required standards of the profession. Further work is now necessary to explore how 'user-friendly' the tool is for healthcare educators to code their own training interventions, and to identify any training and support needs and develop those as necessary. 


\section{References}

1. Health \& Care Professions Council. Continuing professional development (CPD) and HCPC registration. 2017. [Accessed August 4 ${ }^{\text {th }}$, 2017]. http://www.hcpcuk.org/registrants/cpd/intro/

2. Tran D, Tofade T, Thakkar N, Rouse M. US and International Health Professions' Requirements for Continuing Professional Development. Am J Pharm Educ. 2014; 78(6): 129.

3. NHS England Five Year Forward View. 2014

4. Miller GE. The assessment of clinical skills/competence/performance. Acad Med. 1990; 65 (9) Suppl: S63-7.

5. Michie S, van Stralen M, West R. The Behaviour Change Wheel: a new method for characterising and designing behaviour change interventions. Implementation Science. 2011; $6: 42$.

6. Steinmo S, Fuller C, Stone SP, Michie S. Characterising an implementation intervention in terms of behaviour change techniques and theory: the 'Sepsis Six' clinical care bundle. Implementation Science. 2015; 10:111.

7. Oberjé, EJM., Dima, AL., Pijnappel, FJ., Prins, JM., de Bruin, M. Assessing treatment-asusual provided to control groups in adherence trials: Exploring the use of an open-ended questionnaire for identifying behaviour change techniques. Psychology \& Health. 2015; 30 (8): 897-910.

8. Michie S, Richardson M, Johnston M, Abraham C, Francis J, Hardeman W, Eccles MP, Cane J, Wood CE. The behavior change technique taxonomy (v1) of 93 hierarchically 
clustered techniques: Building an international consensus for the reporting of behavior change interventions. Ann Behav Med. 2013; 46 (1):81-95.

9. Chih-Hsiang Y, Maher JP, Conroy DE. Implementation of Behavior Change Techniques in Mobile Applications for Physical Activity. American Journal of Preventive Medicine. 2015; 48 (4): $452-455$.

10. Kahwati L, Viswanathan M, Golin CE, Kane H, Lewis M, Jacobs S. Identifying configurations of behavior change techniques in effective medication adherence interventions: a qualitative comparative analysis. Systematic Reviews. 2016; 5:83.

11. Byrne-Davis LMT, Bull ER, Burton A, Dharni N, Gillison F, Maltinsky W, Mason C, Sharma N., Armitage CJ, Johnston M, et al. How behavioural science can contribute to health partnerships: the case of The Change Exchange. Globalization and Health. 2017; 13:30.

12. Bull ER, Mason C, Domingos Junior F, Santos LV, Scott A, Ademokun D, Simião Z, Oliver WM, Joaquim FF, Cavanagh SM. Developing nurse medication safety training in a health partnership in Mozambique using behavioural science. Globalization and Health. $2017 ; 13: 45$

13. Byrt T, Bishop J, Carlin JB. Bias, prevalence and kappa. Journal of Clinical Epidemiology. 1993; 46 (5):423-429.

14. Algar K, Woods RT, Windle,G. Measuring the quality of life and well- being of people with dementia: A review of observational measures. Dementia. 2014 [published online 24.6.14]. DOI: $10.1177 / 1471301214540163$.

15. Schön, DA. The Reflective Practitioner: How Professionals Think in Action. London: Temple Smith. 1983. 


\section{Illustrations and Tables}

Table 1: Phase one and two inter-rater reliability figures for course observations

\begin{tabular}{|c|c|c|c|c|c|c|c|}
\hline \multirow{2}{*}{$\begin{array}{l}\text { Inter-rater } \\
\text { Reliability } \\
\text { Statistic }\end{array}$} & \multicolumn{2}{|c|}{$\begin{array}{l}\text { Course One (One- } \\
\text { day course) }\end{array}$} & \multicolumn{3}{|c|}{$\begin{array}{l}\text { Course Two (Two-day } \\
\text { course) }\end{array}$} & \multicolumn{2}{|c|}{$\begin{array}{l}\text { Course Three } \\
\text { (Two-day course) }\end{array}$} \\
\hline & $\begin{array}{l}\text { Phase } \\
\text { One }\end{array}$ & $\begin{array}{l}\text { Phase } \\
\text { Two }\end{array}$ & $\begin{array}{l}\text { Phase } \\
\text { One } \\
\text { (Day } \\
\text { 1) }\end{array}$ & $\begin{array}{l}\text { Phase } \\
\text { Two } \\
\text { (Day 2) }\end{array}$ & $\begin{array}{l}\text { Phase } \\
\text { Two } \\
\text { (Day 1) }\end{array}$ & $\begin{array}{l}\text { Phase } \\
\text { One } \\
\text { (Day 2) }\end{array}$ & $\begin{array}{l}\text { Phase } \\
\text { Two } \\
\text { (Day 1) }\end{array}$ \\
\hline Cohen's Kappa & 0.88 & 0.83 & 0.86 & 0.75 & 0.84 & 0.89 & 0.89 \\
\hline $\begin{array}{l}\text { Prevalance And } \\
\text { Bias Adjusted } \\
\text { Kappa (PABAK) }\end{array}$ & 0.92 & 0.87 & 0.90 & 0.81 & 0.87 & 0.91 & 0.91 \\
\hline
\end{tabular}


Figure 1. Summary of methods

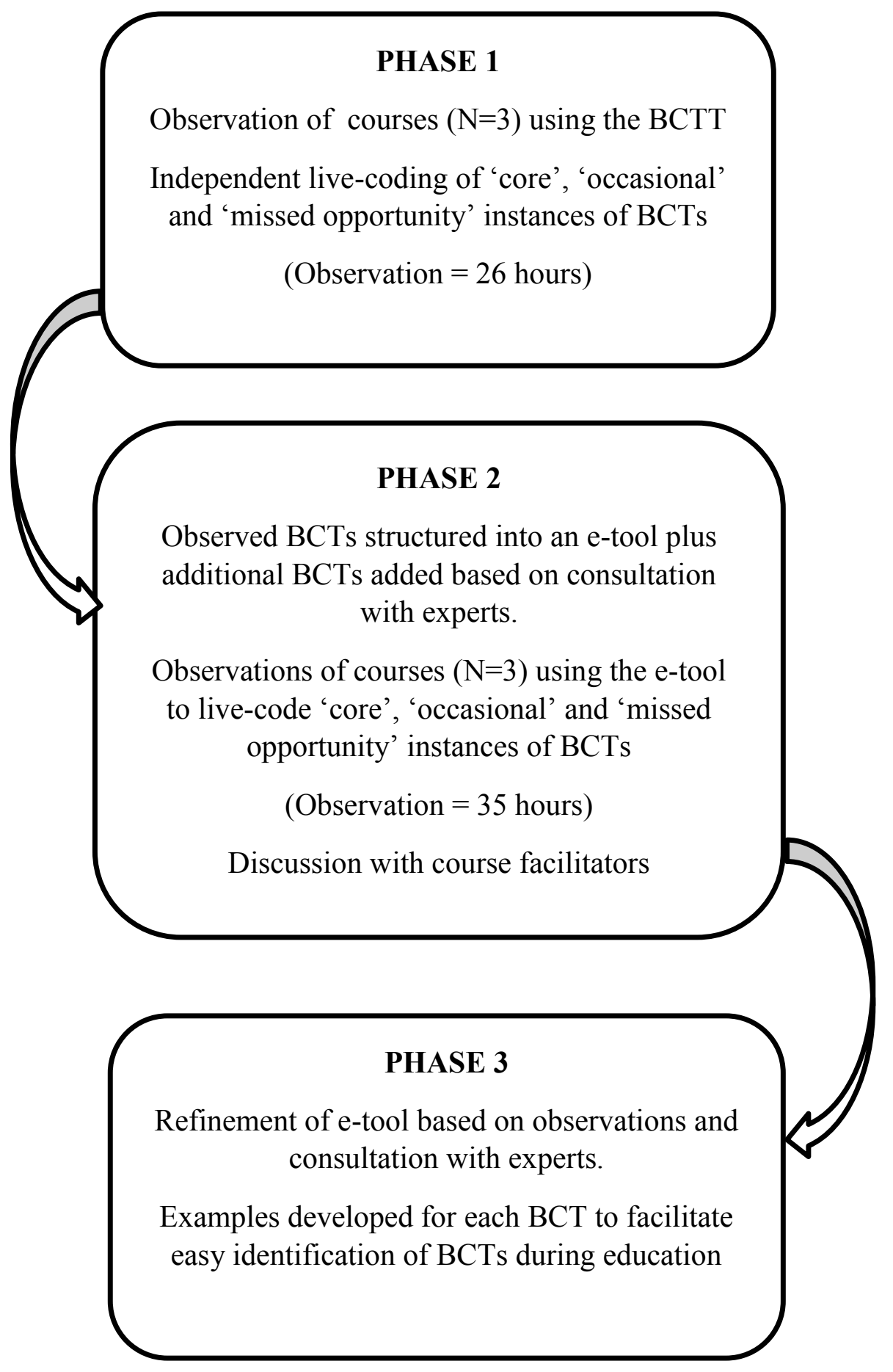


Figure 2. Observed use of BCTs in three medical CPD courses (both occasional and core)

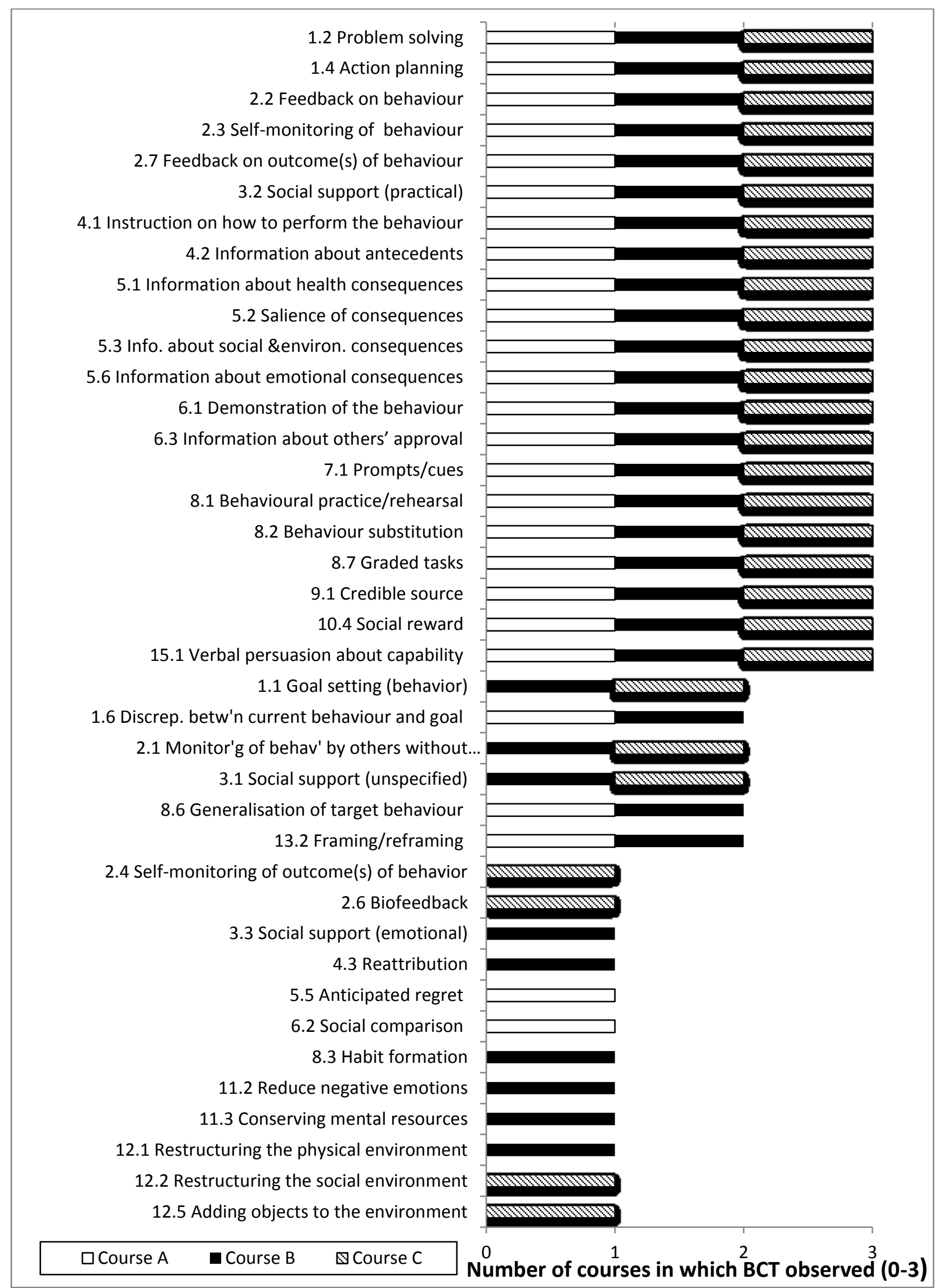


Figure 3. Observed BCT 'missed opportunities' in three medical CPD courses

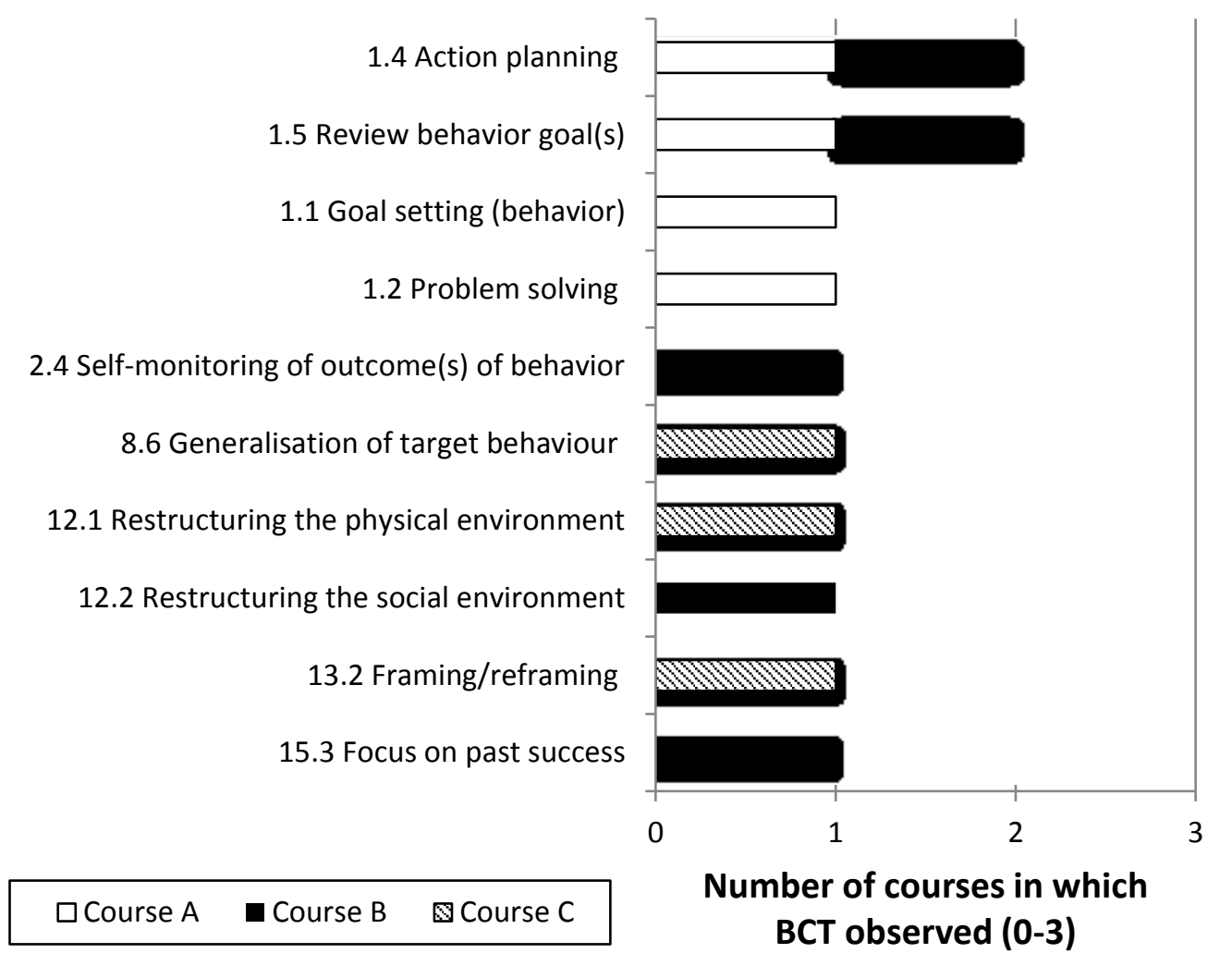

